\title{
Ileosigmoid Knot-Unveiling the Diagnostic Dilemma with Radiologic Signs: A Case Report
}

\author{
Poonam Hegde ${ }^{1}$ Rupa Ananthasivan ${ }^{1}$ Sudarshan Rawat ${ }^{1} \quad$ Pooja G. Patil ${ }^{1}$ Bhavana Girishekar ${ }^{1}$ \\ Mrinal Matish ${ }^{1}$
}

1Department of Radiology and Imaging, Manipal Hospitals, Bangalore, Karnataka, India

\begin{abstract}
Address for correspondence Poonam Parameshwar Hegde, MBBS, DMRD, DNB (Radio-diagnosis), Department of Radiology and Imaging, Manipal Hospitals, HAL Old Airport Road, Bangalore 560017, Karnataka, India (e-mail: hegdepoonam235@gmail.com).
\end{abstract}

\begin{abstract}
lleosigmoid knot (ISK) is an unusual and serious condition that is known to pose a diagnostic dilemma due to overlapping features of acute closed-loop intestinal obstruction. Early diagnosis and intervention are of immense importance in this condition because they can prove life threatening with rapid progression to gangrene of both the ileum and sigmoid colon. We report a case of a 27 -year-old man who presented 2 days post-appendicectomy procedure with acute abdominal pain, abdominal distention, and hypovolemic shock. Initial evaluation with ultrasonography (USG) and abdominal $\mathrm{X}$-ray was performed, which revealed pleural effusion, ascites, and an ahaustral dilated large bowel loop in the right upper abdomen with convergence in the left lower

Keywords

- ileosigmoid knot

- intestinal obstruction

- bowel gangrene

- whirl sign abdomen. Further imaging with computed tomography revealed closed-loop obstruction of the sigmoid colon with beaked appearance of its afferent and efferent limbs in the pelvis. There was associated whirling or knotting of the ileum around the sigmoid pedicle along with features of mesenteric ischemia. Emergency laparotomy was performed, which revealed ISK with gangrene of the ileal and large bowel loops.
\end{abstract}

\section{Introduction}

Ileosigmoid knot (ISK), also called compound volvulus or double volvulus, is a rare cause of acute closed-loop bowel obstruction. ${ }^{1,2}$ In this condition, the ileum is seen encircling around the sigmoid pedicle and forming a knot that can lead to varying degrees of bowel obstruction and vascular compromise. ${ }^{1}$ This condition is known to occur more often among the Asian, African, and the Middle Eastern population. ${ }^{1}$ The etiology is multifactorial. We highlight the radiologic signs of ISK with emphasis on computed tomography (CT) scan features that can assist in early diagnosis and management of this serious condition.

\section{Case Presentation}

A 27-year-old man presented to the adult emergency department with diffuse abdominal pain, abdominal distention, and inability to pass stools and flatus. On general examination, the patient was conscious and oriented. There were tachypnea with a respiratory rate of 24 breaths/min, tachycardia with

received

August 30, 2018

accepted after revision

October 28, 2018 low volume pulse rate of 122 beats/min, and blood pressure 90/60 $\mathrm{mm} \mathrm{Hg}$. On abdominal examination, there were diffuse tenderness, guarding, and rigidity with abdominal distention. Laboratory investigations revealed raised serum creatinine and blood urea nitrogen. The total white blood cell (WBC) count was $4,390 / \mathrm{mm}^{3}$ with mildly raised neutrophils (85\%). A previous 2-day history of diffuse abdominal pain, for which the patient was evaluated and underwent laparoscopic appendicectomy at an outside hospital, was obtained.

In view of abdominal sepsis, the patient was shifted to medical intensive care unit for mechanical ventilation. Specific organ support and dialysis were started. A bedside ultrasonography (USG) of the abdomen was performed, which revealed bilateral pleural effusions, moderate ascites, and multiple dilated bowel loops. Supine X-ray of the abdomen (-Fig. 1) showed a dilated gas-filled loop of large bowel occupying the right mid and lower quadrants with predominant ahaustral pattern of bowel dilatation. It was seen forming a closed loop with convergence in the pelvis identifying it as sigmoid colon. No rectal gas shadow was 


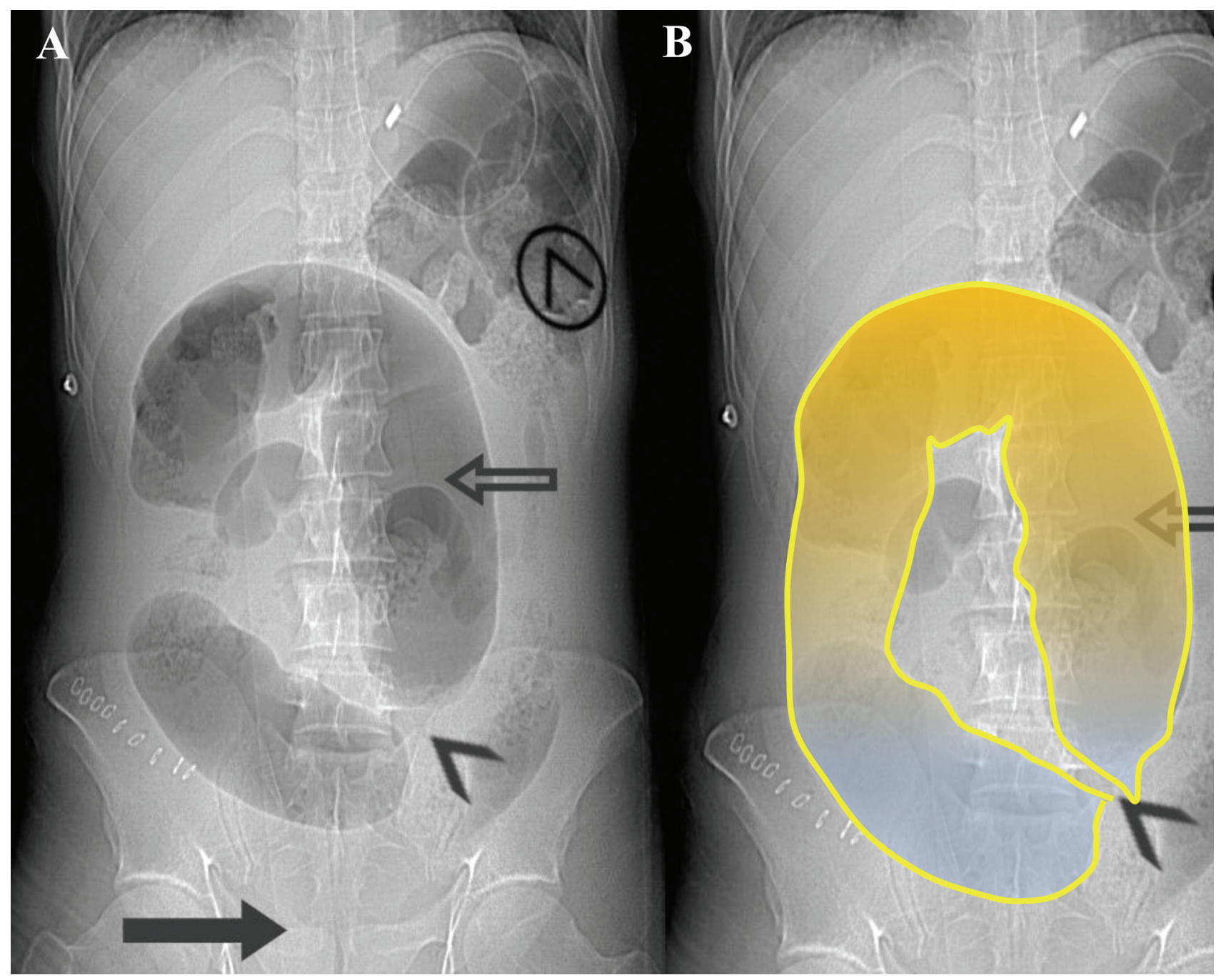

Fig. 1 (A) Supine X-ray of the abdomen shows dilated ahaustral large bowel loop in right abdominal quadrant (outlined arrow). It is forming closed loop with convergence in pelvis (arrowhead). Absent rectal gas shadow (solid black arrow). Few gas and feces filled large bowel loops in left hypochondrium (circled arrowhead). (B) Line diagram delineating the dilated ahaustral loop of colon (outlined arrow) with convergence in the pelvis (arrowhead).

identified. The possibility of closed-loop large bowel obstruction and sigmoid volvulus was considered at this time.

A contrast-enhanced multidetector CT scan of the abdomen and pelvis was performed on GE LightSpeed 64-slice Multidetector Volume CT scanner (Milwaukee, Wisconsin, United States). The 5-mm thin axial sections were acquired in the plain, arterial, venous, and delayed contrast phases. The abdominal CT examination was performed at $120-\mathrm{kV}$ tube voltage and 220-mAs tube current. Intravenous iodinated contrast (Omnipaque, GE Healthcare, Shanghai, China) was given at a concentration of $300 \mathrm{mg}$ iodine $/ \mathrm{mL}$ at the rate of 3 $\mathrm{mL} / \mathrm{s}$ up to a total dose of $100 \mathrm{~mL}$. The scans were studied in the axial and multiplanar mode.

CT demonstrated a markedly dilated loop of the sigmoid colon measuring up to $7 \mathrm{~cm}$ in maximum luminal diameter occupying the right half of the abdomen ( - Fig. 2A). The walls of the sigmoid colon appeared thinned out with loss of haustral pattern (-Fig. 2A). There was twisting of the mesentery and sigmoid mesocolon along with the bowel giving the typical "whirl sign" (-Fig. 2B). The afferent and efferent limbs of the sigmoid colon were seen converging into the pelvis causing beaking ( - Fig. 3). Few ileal loops were seen wrapping around and encompassing the narrow sigmoid pedicle, associated with few prominent proximal small bowel loops. This along with the twisting of the mesentery produced the whirled knot ( - Fig. 4$)$. There was relative medial deviation of the cecum and distal descending colon with pointed appearance of their medial end ( - Fig. 5) marking the lateral margins of the volvulus. Few of the small bowel loops also revealed mild wall edema ( - Fig. 6A). In addition, there were a few prominent small bowel loops that revealed intramural air, suspicious for pneumatosis ( - Fig. 6 B $)$. Mild pneumoperitoneum, moderate ascites ( $\mathbf{F}$ Fig. $\mathbf{6 B}$ ), and mild bilateral pleural effusion were identified.

At this point, the diagnosis of closed-loop large bowel obstruction secondary to sigmoid volvulus, complicated with mesenteric and bowel ischemia, was made. In view of CT features of wrapping of the ileum around the sigmoid colon ( - Fig. 7), the suspicion of ISK was also raised.

The patient underwent an emergency laparotomy. On exploration, the distal ileal loop was seen forming a tight constriction around the sigmoid colon with gangrenous changes involving both the sigmoid colon and ileal segments 

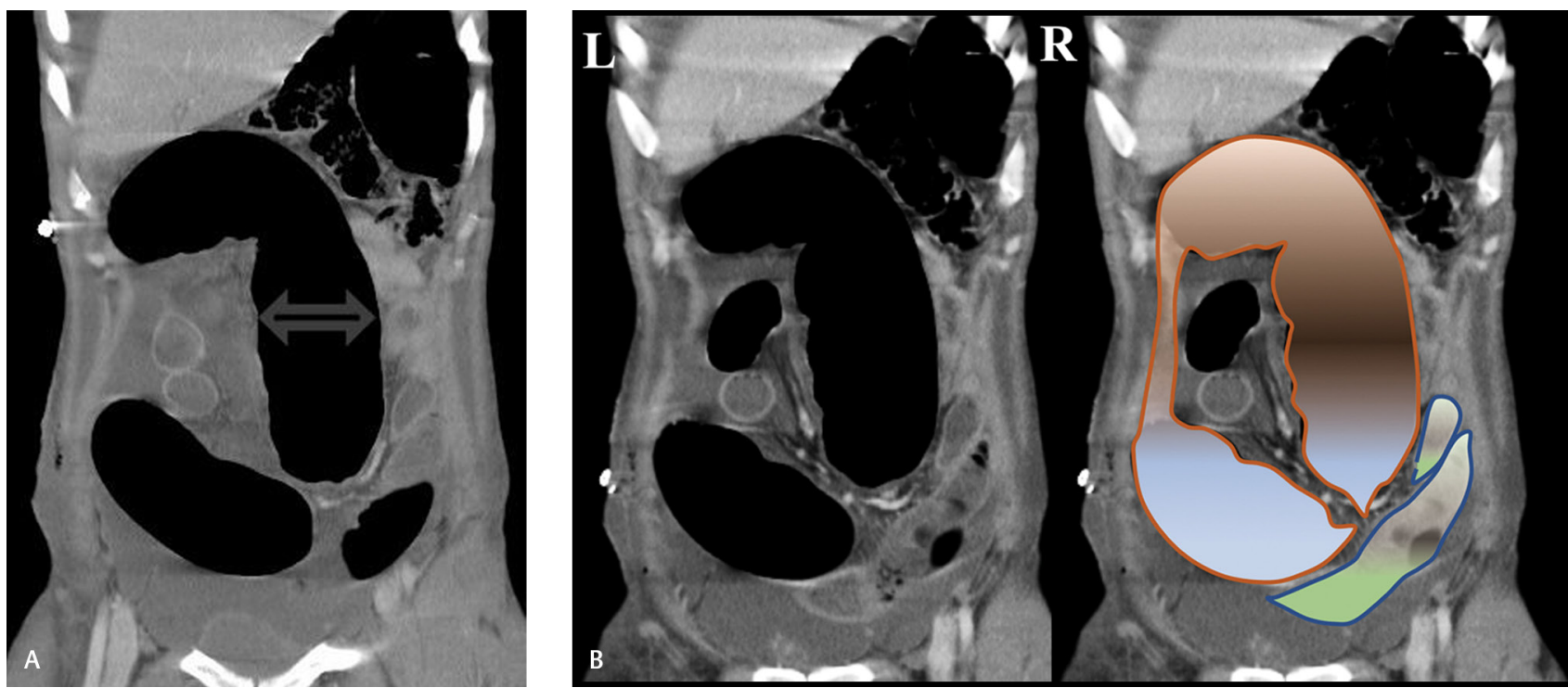

Fig. 2 Coronal CT images in venous phase: (A) Dilated sigmoid colon with loss of haustral pattern and thinned-out wall (double-headed arrow). (B) Left panel (L) shows the CT finding, and the corresponding schematic line diagram in right panel (R) shows convergence of sigmoid loop in pelvis (orange outline), with twisting of mesentery and mesocolon and adjacent ileal segment (blue outline) on left side of sigmoid.

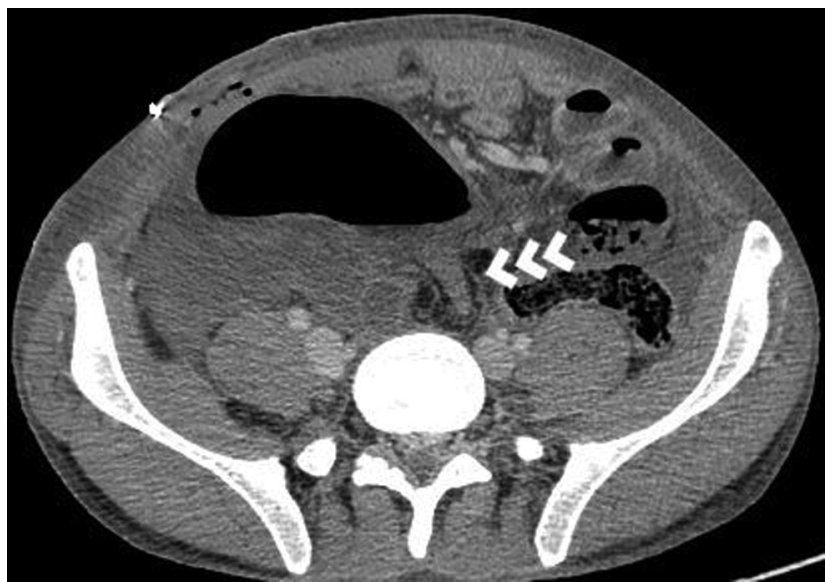

Fig. 3 Axial CT image in venous phase: Convergence of afferent and efferent loops of sigmoid colon to form closed loop giving "beaked appearance" (triple white arrowhead).

(-Fig. 8). Resection of ISK along with the distal ileum and sigmoidectomy was performed. This was followed by end ileostomy and end descending colostomy. The patient received postoperative support with analgesia and ventilation, following which he improved and was extubated. Postoperatively he was hemodynamically stable and was discharged.

\section{Discussion}

ISK is a well-known yet rare entity presenting as acute bowel obstruction. In this condition, the ileal loops are seen to twist around the sigmoid colon over a narrow pedicle, forming constriction to variable extents. ${ }^{1,2}$ This knotting of the ileum around the sigmoid colon is accompanied by the whirling of the adjacent mesentery and mesocolon that is responsible for the ischemic insult, which sets in early in cases of ISK. ${ }^{1}$ The intraoperative findings often typically consist of gangrenous changes involving the ileal segments as well as the sigmoid

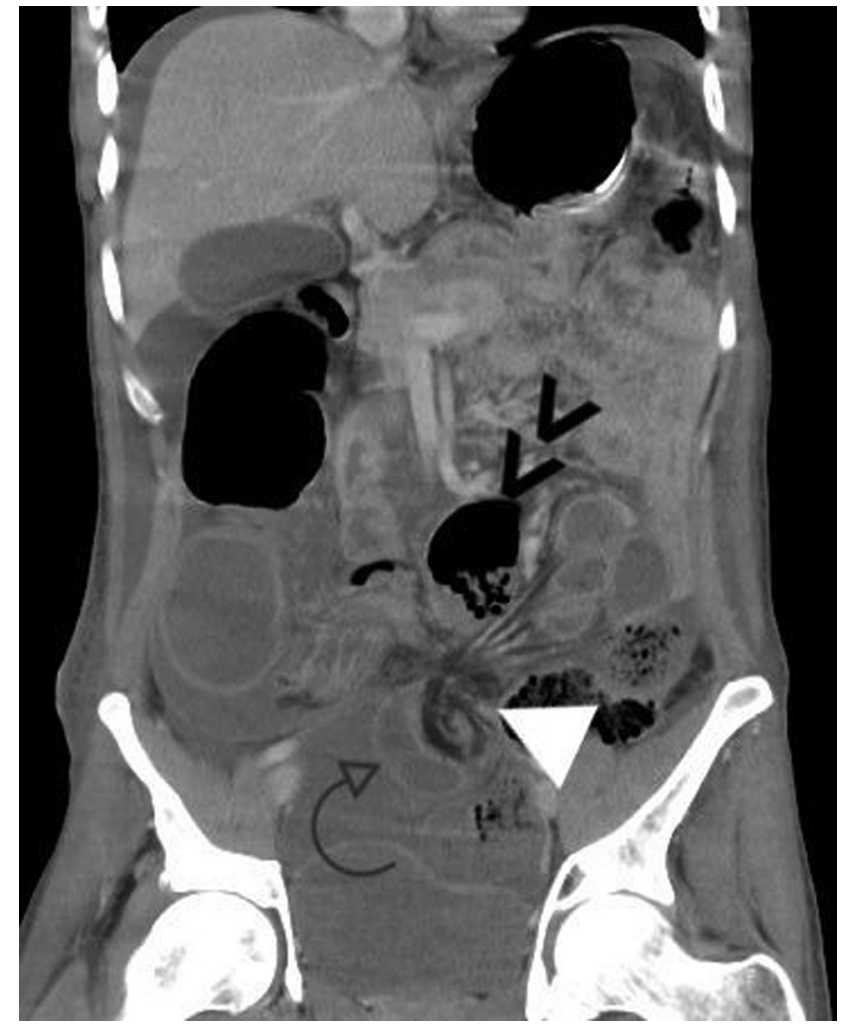

Fig. 4 Coronal CT image in venous phase: lleal loops winding across narrow sigmoid pedicle at transition zone forming a whirl/knot along with twisting of the mesentery giving the classic "whirl sign" (white arrowhead). Sigmoid colon (double black arrowhead) and ileal loop (curved arrow).

colon with nongangrenous intervening the large bowel segments. However, in few cases, the large bowel is known to be affected to variable degrees with involvement of longer large bowel segments. ${ }^{1,3}, 4$ The etiologic factors responsible for this condition have been postulated to be a long small bowel mesentery associated with excessively mobile small 


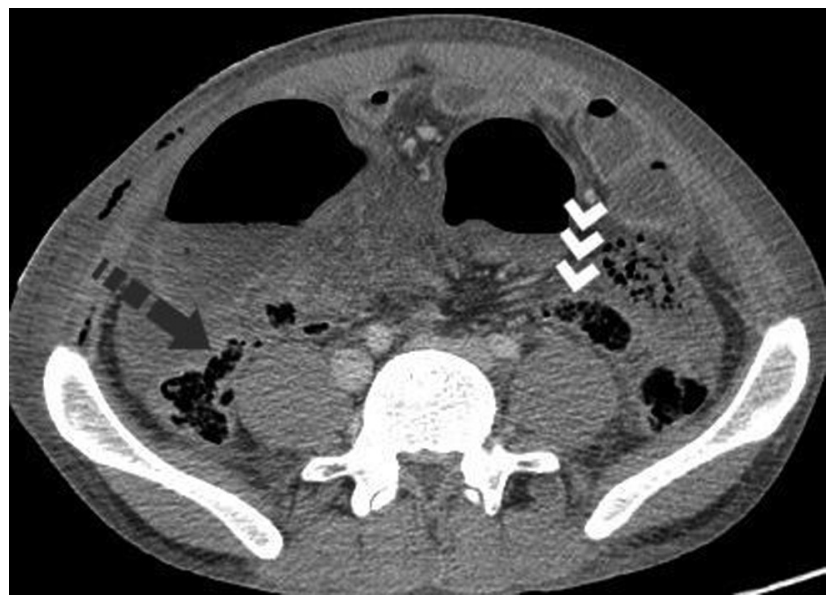

Fig. 5 Axial CT image in venous phase: Medial deviation of cecum (dotted bold gray arrow) and distal descending colon (triple white arrowhead) with pointed appearance of their medial borders.

bowel loops and increased redundancy of sigmoid colon over a long mesocolon, which allows easy twisting around a narrow base. The consumption of high bulk diet in presence of relatively empty small bowel has also been considered as a predisposing factor. ${ }^{3,5,6}$ This condition is known to show higher incidence of occurrence among the male population in the fourth decade. ${ }^{7}$

The patient presents with symptoms of acute abdominal pain and intestinal obstruction. ${ }^{1,6-8}$ Complications include hypovolemic shock, peritonitis, sepsis, and bowel gangrene. ${ }^{1,7}$

ISK has been categorized into four types by Alver:

- Type I-The most common type in which the ileum acts as an active component encircling around the passive sigmoid colon.

- Type II-The sigmoid colon being the active component wraps itself around the ileum forming the knot.

- Type III-In this type, the ileocecal junction as a whole behaves as the active component, knotting around the sigmoid colon.

- Type IV-Undetermined type.

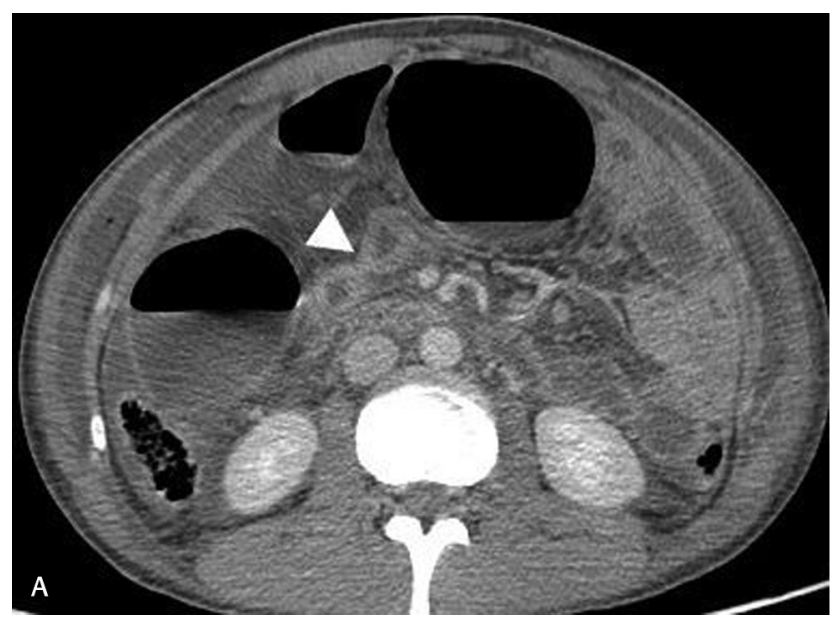

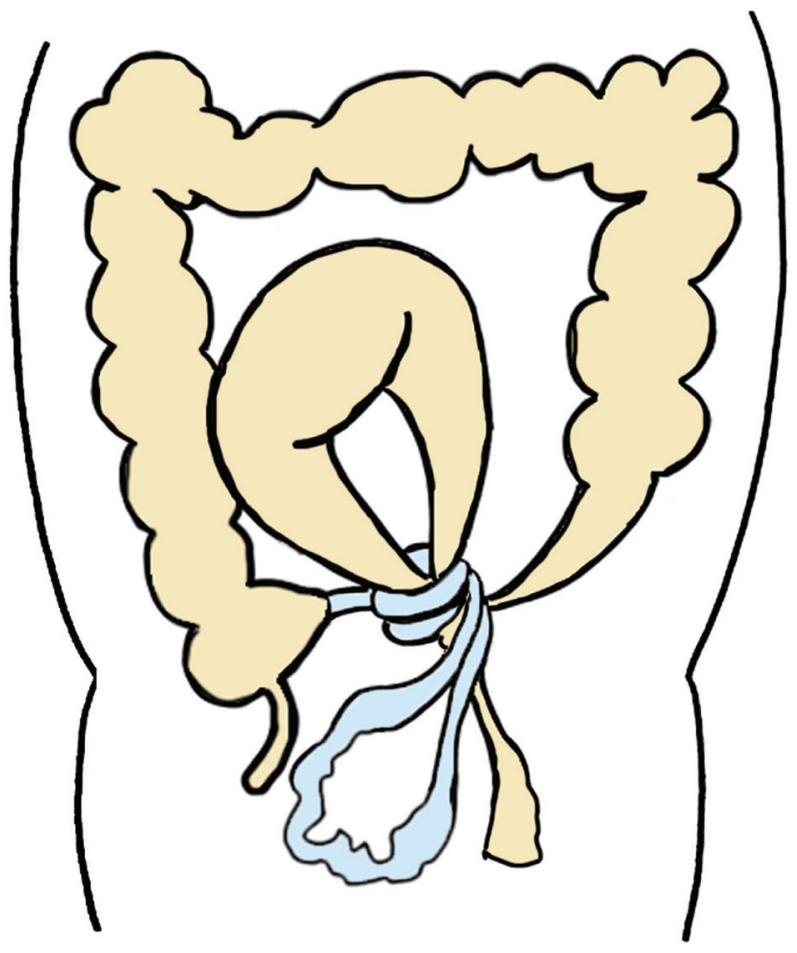

Fig. 7 Schematic drawing showing anatomic relationship of colon (orange color) and ileum (blue color) in ISK, wherein the ileal loops are seen twisting around a narrow base of the dilated sigmoid loop. Medial deviation with medial beaking of descending colon and cecum is demonstrated.

Types I and II are further classified into subtypes A and B, wherein subtype $A$ involves torsion of the active component around the passive component in a clockwise direction and vice versa, that is, anticlockwise in subtype B. ${ }^{1,8,9}$

ISK has continued to remain a diagnostic dilemma with relatively low incidence of preoperative diagnosis, especially due to inconsistent and overlapping radiologic signs. ${ }^{2}$ Plain abdominal radiograph findings often mimic sigmoid volvulus with air-filled dilated sigmoid colon in the right upper quadrant with convergence in the pelvis. The classic

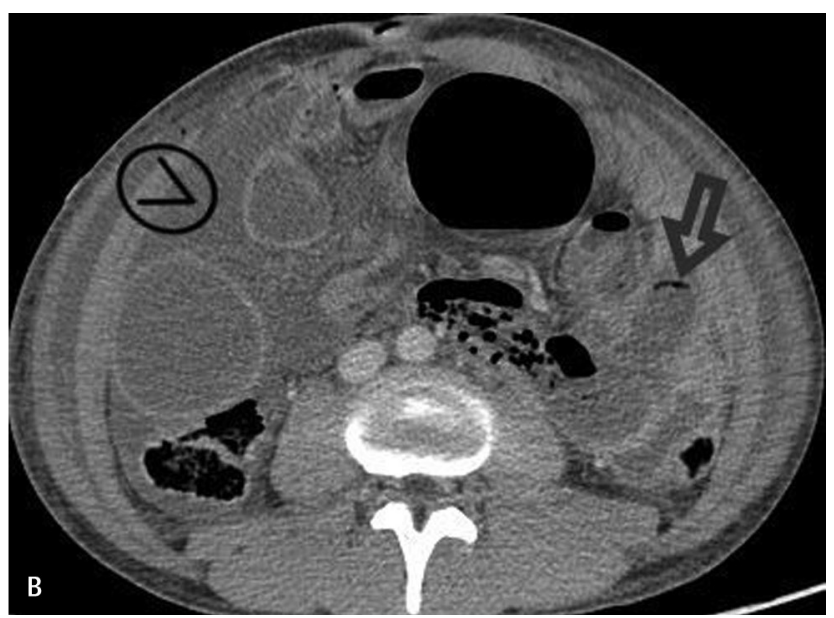

Fig. 6 Axial CT images in venous phase: (A) Small bowel wall edema (white arrowhead). (B) Ascites (circled arrowhead) and foci of intramural air in small bowel (outlined arrow). 


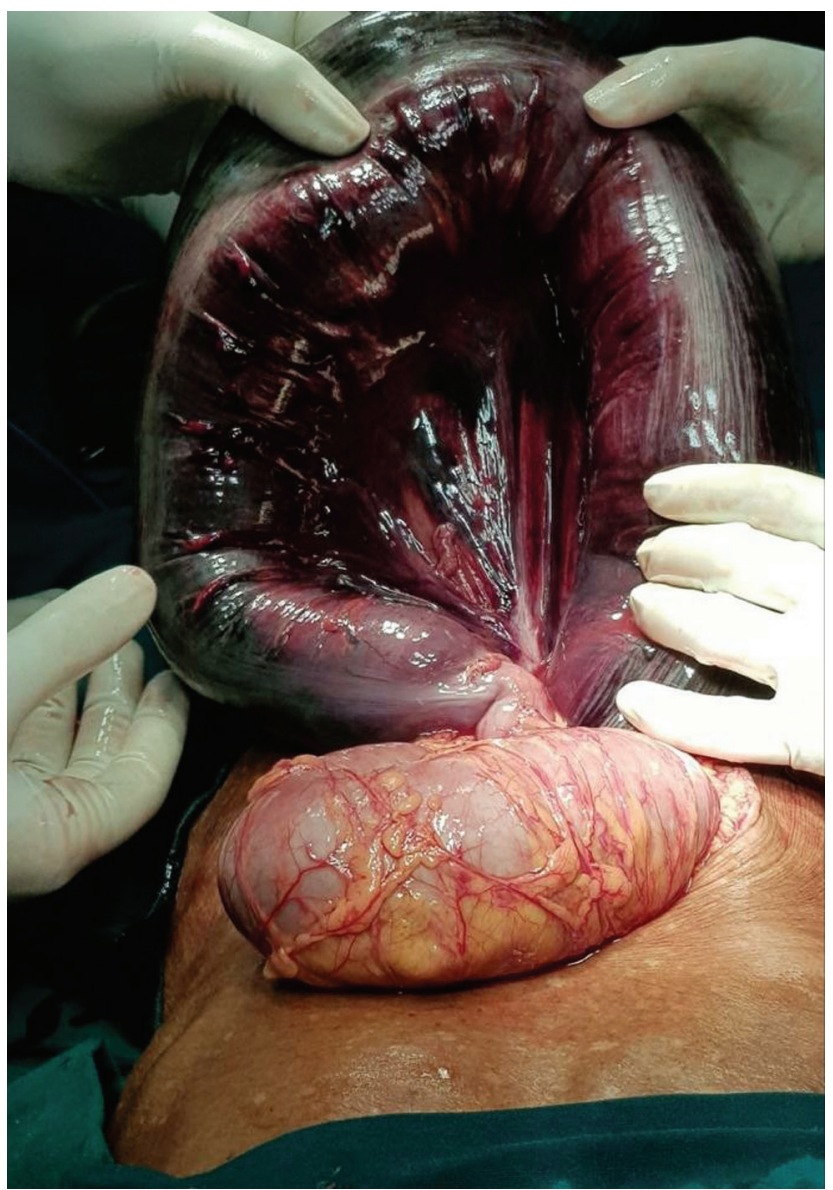

Fig. 8 Intraoperative surgical photograph showing dilated sigmoid colon with gangrene secondary to lleosigmoid knotting.

features of double closed-loop obstruction with dilated sigmoid colon to the right and dilated small bowel loops in the left quadrant is an infrequent finding., ${ }^{4,10,11}$ USG shows dilated bowel loops and other ancillary findings such as ascites and pleural effusion. It can aid in guided diagnostic ascitic tap that often reveals hemorrhagic fluid.

CT scan features can improve the preoperative diagnostic accuracy and help timely intervention. CT scan reveals the typical "whirl sign" created by the torsion of the intestine and mesocolon., ${ }^{2,12}$ The presence of the whirl in several contiguous CT slices as compared with sigmoid volvulus is seen. ${ }^{2,8}$ The sigmoid colon shows dilated, ahaustral, and thinned-out poorly enhancing walls with ileal segments winding around the sigmoid pedicle to produce a knot. ${ }^{13}$ Convergence of the afferent and efferent limbs of the sigmoid along with twisted mesentery and mesenteric vessels toward the pelvis produces "beaking." ${ }^{4,6}$ Concurrent ischemic changes in the ileal loops and sigmoid colon are highly suspicious for ISK. Bowel ischemia due to strangulation may be complicated with pneumatosis and pneumoperitoneum. Another distinct CT feature of ISK includes medial deviation with pointed appearance of the cecum and distal descending colon. The parietal peritoneum in the left paracolic gutter is pulled toward the knot, and the dilated ileum lying between the descending and proximal sigmoid colon causes mass effect that is likely to be the cause for the medial deviation. The cecum is also pulled medially due to the stretching of the terminal ileum., ${ }^{2,8,14}$

ISK is a surgical emergency and warrants an exploratory laparotomy. Unwinding of the knot may be attempted in cases of retained vascular supply and viable bowel loops. However, in most cases untwisting is difficult, and when bowel gangrene is demonstrated intraoperatively, resection of the ISK and the gangrenous segments is recommended followed by primary anastomosis. ${ }^{10}$

\section{Conclusion}

ISK is an unfamiliar and serious cause of intestinal obstruction that is known to rapidly progress to bowel ischemia and gangrene. We present here a case of ISK that was accurately diagnosed preoperatively with the use of various imaging modalities and radiologic signs, and particularly the CT scan findings aiding in early intervention, hence reducing the incidence of mortality and morbidity.

\section{Conflict of Interest}

None declared.

\section{References}

1 Alver O, Oren D, Tireli M, Kayabaşi B, Akdemir D. Ileosigmoid knotting in Turkey. Review of 68 cases. Dis Colon Rectum 1993;36(12):1139-1147

2 Lee SH, Park YH, Won YS. The ileosigmoid knot: CT findings. AJR Am J Roentgenol 2000;174(3):685-687

3 Akgun Y. Management of ileosigmoid knotting. Br J Surg 1997;84(5):672-673

4 Atamanalp SS, Oren D, Başoğlu M, et al. Ileosigmoidal knotting: outcome in 63 patients. Dis Colon Rectum 2004;47(6):906-910

5 Kakar A, Bhatnagar BN. Ileo-sigmoid knotting: a clinical study of 11 cases. Aust N Z J Surg 1981;51(5):456-458

6 Machado NO. Ileosigmoid knot: a case report and literature review of 280 cases. Ann Saudi Med 2009;29(5):402-406

7 Shepherd JJ. Ninety-two cases of ileosigmoid knotting in Uganda. Br J Surg 1967;54(6):561-566

8 Baheti AD, Patel D, Hira P, Babu D. Ileosigmoid knot: a case report. Indian J Radiol Imaging 2011;21(2):147-149

9 Atamanalp SS, Ozturk G, Aydinli B, et al. A new classification for ileosigmoid knotting. Turk J Med Sci 2009;39:541-545

10 Mandal A, Chandel V, Baig S. Ileosigmoid knot. Indian J Surg 2012;74(2):136-142

11 Hirano Y, Hara T, Horichi Y, et al. Ileosigmoid knot: case report and CT findings. Abdom Imaging 2005;30(6):674-676

12 Catalano O. Computed tomographic appearance of sigmoid volvulus. Abdom Imaging 1996;21(4):314-317

13 Sangwan M, Sangwan V, Garg MK, Mutreja J, Singla D, Gautam D. Ileosigmoid knotting: a rare case report with review of literature. J Surg Case Rep 2015;2015(5):rjv051

14 Tamura M, Shinagawa M, Funaki Y. Ileosigmoid knot: computed tomography findings and the mechanism of its formation. ANZ J Surg 2004;74(3):184-186 